\title{
Families Coping: Effective Strategies for You and Your Child
}

Erica Frydenberg

ACER Press, 2015, 96 pp., \$39.95 (AU paperback), ISBN: 978-1-74-286253-8.

doi: $10.1017 /$ edp. 2015.2

Parenting practices have a major influence on child development and behaviour. Many new parents are motivated to develop and improve their parenting skills so that they can give their children the best possible start in life. Families Coping: Effective Strategies for You and Your Child, a new book published by ACER Press, aims to teach parents how to raise resilient and resourceful children who are able to effectively negotiate relationships with parents, siblings, peers and teachers. The book can be used as a selfhelp manual for parents or as an instructor-led parenting program. It brings together two theoretical frameworks, a skills-based humanistic positive parenting approach and the transactional model of stress and coping, and emphasises that positive parent-child interactions are an essential component of successful parenting. The attention given to enhancing the quality of the parent-child relationship is part of a burgeoning trend in parenting programs and is well supported by research evidence. In their meta-analytic review of 77 evaluations of parent training programs, Wyatt Kaminski, Valle, Filene, and Boyle (2008) found that programs that focused on providing parents with the skills to improve the parent-child relationship were more effective. Families Coping: Effective Strategies for You and Your Child provides a clear picture of what positive parent-child interactions look like in practice and highlights how these interactions will benefit families during the childhood years and beyond.

The book addresses parents of young children aged between 4 and 8. It covers topics such as the positive psychology of parenting, coping with stress in the family, everyday worries and anxieties of children, listening to children, collaborative problem-solving, and mindfulness-based parenting practices. Given that parental consistency is reliably associated with better outcomes for children, it might be useful to devote more space to this subject in future editions of the text.

The book is written in a friendly and engaging style and is well supported by diagrams and activities for parents. The activities are designed to assist parents to recognise both their own and their children's needs and to develop the core skills of effective communication, including reflective listening skills. One of the main strengths of the book is that it frequently requires parents to practise the skills with their children as they are being learned. The book also promotes thoughtful parenting by encouraging parents to continually reflect on their interactions with their children. The importance of reflective parenting is further reinforced by the concluding message of the book, which is that mindful parenting, or bringing mindful attention directly to parent-child interactions, moment by moment (Sawyer-Cohen \& Semple, 2010), can improve parent-child relationships and promote healthy child development.

The book will be an invaluable resource for those who are new to parenting and for those who want a refresher. The book will also provide educational and developmental 
psychologists with a comprehensive review of the foundational skills of effective parenting within the context of the contemporary positive psychology movement.

\author{
Dr Michelle Andrews \\ The Educational Psychology Centre, Melbourne, Australia \\ Disclaimer: The author has previously worked at the University of Melbourne with \\ Erica Frydenberg.
}

\title{
References
}

Sawyer Cohen, J., \& Semple, R. (2010). Mindful parenting: A call for research. Journal of Child and Family Studies, 19, 145-151.

Wyatt Kaminski, J., Valle, L., Filene, J., \& Boyle, C. (2008). A meta-analytic review of components associated with parent training program effectiveness. Journal of Abnormal Child Psychology, 36, 567-589.

\section{Therapeutic Metaphors for Children and the Child Within (Second Edition)}

Joyce C. Mills and Richard J. Crowley

Routledge New York, 2014, 215 pp., \$52.68 (AU paperback) IBSN: 978-0-415-70810-4.

doi:10.1017/edp.2015.3

Therapeutic Metaphors for Children and the Child Within is best for those working with children and adolescents who are interested in counselling, play therapy and seeking alternatives in counselling settings.

Mills and Crowley draw upon the teachings from the late psychiatrist Dr Milton $\mathrm{H}$. Erikson (1901-1980), who specialised in hypnosis, family therapy, solution-focused brief therapy and neuro-linguistic programming.

The main concept of this book is the use of symbolic language of metaphor and storytelling in counselling. It is well regarded in both eastern and western cultures and documented by Kopp (1971) that the 'use of metaphor is a primary vehicle for teaching' (p. 6).

The book is broken into four main parts. Parts I and II (Chapters 1-6) walk through the elements of metaphor and how to create stories with the client. It is apparent that Parts I and II are practically the same book that was released originally in 1986, as the case studies presented have dated pop culture references (e.g., of the recent release of E.T. the Extra Terrestrial).

A lot of research has taken place in the past 30 years; it would have been in the author's best interest to include and acknowledge some of the advances of science (especially in neuroscience) and other counselling techniques and interventions that can also help explain and illustrate the functional use of metaphors (and vice versa). This could have been achieved, as some of the case studies presented used metaphor in such a way that parallels could be drawn to address CBT concepts in creative and illustrative ways and not just constrained to the methods we are familiar with from typical psychological training.

Part III (Chapters 7-8) of this text is where the applications of metaphor are demonstrated in a much more succinct manner and illustrate the potential effectiveness for 